\title{
REFLOW OF NON-CIRCULAR NANO-PILLARS TO FABRICATE NANO-SCALE SOLID IMMERSION LENSES
}

\author{
Myun-Sik Kim, ${ }^{1, *}$ Ethan Keeler, ${ }^{2}$ Skyler Rydberg, ${ }^{2}$ Wataru Nakagawa, ${ }^{2}$ Gaël Osowecki, ${ }^{1}$ \\ Toralf Scharf, ${ }^{1}$ and Hans Peter Herzig ${ }^{1}$ \\ ${ }^{1}$ Optics \& Photonics Technology Laboratory, Ecole Polytechnique Fédérale de Lausanne \\ (EPFL), Breguet 2, 2000 Neuchâtel, Switzerland \\ ${ }^{2}$ Electrical and Computer Engineering Department, Montana State University, P.O. Box \\ 173780, Bozeman, Montana 59717-3780, USA \\ *myunsik.kim@epfl.ch
}

\begin{abstract}
We investigate the reflow of non-circular, i.e., triangular and square, shaped pillars to fabricate nano-scale solid immersion lenses (SILs). Electron beam lithography (EBL) and thermal reflow are the core of the fabrication process. For the optical characterization at $\lambda=642 \mathrm{~nm}$, nano-SILs are replicated on a transparent substrate by soft lithography. The focal spots produced by the nano-SILs show both spotsize reduction and peak-intensity enhancement, which are consistent with the immersion effect.
\end{abstract}

\section{INTRODUCTION}

In 1990, Mansfield et al. proposed a new immersion technique for microscopy, called a solid immersion lens [1]. The SIL increases the numerical aperture [NA $=n \cdot \sin \theta]$ by a factor of $n$ (the refractive index of the SIL), as in liquid immersion. For a long time the fabrication of SILs was limited to macro-size, i.e. millimeter range dimensions, due to the lack of advanced fabrication technologies. The advance of micro- and nano-technology boosts the development of different types of SILs, for instance, diffractive SILs [2] and micro-scale SILs [3-5]. In our previous work, we demonstrated for the first time the fabrication and optical characterization of nano-scale SILs down to subwavelength size [6].

In this work, we diversify the fabrication of nano-SILs by employing non-circular shaped pillars, such as 400-nm square and $600-\mathrm{nm}$ triangular patterns. Standard circular patterns are considered as well. Furthermore, the optimal designs and process conditions are investigated in order to achieve the ideal hemispherical structure. The performance of the fabricated nano-SILs is verified by measuring the intensity distributions of the immersed focal spots, which are compared to a reference non-immersion spot to reveal the diminution of the spot size and the enhancement of the peak intensity.

\section{FABRICATION}

Nano-scale pillars having several shapes are patterned by electron-beam lithography: $450 \mathrm{~nm}$ cylinders, $400 \mathrm{~nm}$ square pillars and $600 \mathrm{~nm}$ triangular pillars, which are shown in the scanning electron microscope (SEM) images of Fig. 1. We investigate two subjects. One is the reflow and the shape transformation of the non-circular pillars. The second is the optimal process conditions needed to produce the desired hemispherical structures after reflow. To do so, we vary the height $[200 \mathrm{~nm}, 250 \mathrm{~nm}, 300 \mathrm{~nm}]$ and the diameter [420 nm or $480 \mathrm{~nm}$ ] of the cylindrical patterns. Variation of the reflow temperature is also considered.
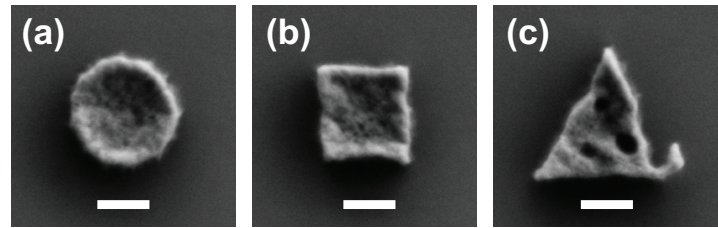

Fig. 1. SEM images of nano-pillars: (a) 450-nm circle, (b) 400-nm square and (c) 600-nm triangle. The scale bars indicate $200 \mathrm{~nm}$.

To pattern a nano-pillar using EBL, an area of approximately $4 \mu \mathrm{m}$ square with a void in the shape of the desired pillar at its center is exposed. Since polymethyl methacrylate (PMMA) is a positive resist, during the development process this area is removed, leaving a freestanding pillar of PMMA. As PMMA is also a thermoplastic, thermal reflow is applied to transform the flat-top pillar into a spherical structure. The glass transition temperature $\left(\mathrm{T}_{\mathrm{g}}\right)$ of PMMA is approximately $110 \sim 120^{\circ} \mathrm{C}$, which mainly depends on the electron beam dose [7]. We heat samples above $\mathrm{T}_{\mathrm{g}}$ by using a hot plate. At $120{ }^{\circ} \mathrm{C}$, shape transformation is not observed except for surface smoothing and rounding of edges. When the reflow temperature is higher than $140{ }^{\circ} \mathrm{C}$, the over-reflow causes widening of the lateral size and a reduction in the height of the SIL. Thus, the optimal reflow temperature is found to be $130{ }^{\circ} \mathrm{C}$, where reflow speed is slow enough for better control compared to higher temperatures. Reflow at $130{ }^{\circ} \mathrm{C}$ for $50 \mathrm{~min}$. leads to the full reflow of the pillars without the symptoms of over flow. Interestingly, both the square and triangular pillars are transformed into a circular shape after reflow. Since the volume of the pillar is very small, the heating to reflow temperature can lead to melting of the outer boundary, and wetting produces the spherical shape. In nano-scale fabrication, it is a challenging task to realize structures without errors (Fig. 1 demonstrates such issues). For example, the circle is slightly elliptic, and the square and the triangle are deformed as well. The reflow and wetting are a good solution to overcome this problem for the fabrication of nano-SILs, which essentially require spherical structures. The pillars are fabricated on silicon substrates to achieve the best resolution in EBL. For optical 
characterization with visible light (at $\lambda=642 \mathrm{~nm}$ ), the reflowed spherical structures, i.e. the SILs, must be replicated on a transparent substrate. Soft lithography using a UV curable polymer (Norland, NOA 61, $\mathrm{n}=1.56$ ) enables replication of the SILs on borosilicate glass cover slips (thickness $=150 \mu \mathrm{m}, \mathrm{n}=1.52$ ). Figure 2 shows SEM images of the replicated nano-SILs on glass substrates.
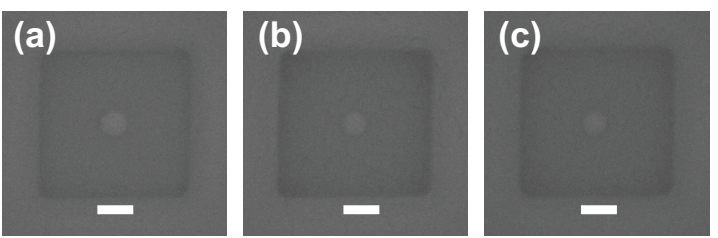

Fig. 2. SEM images of the replicated nano-SILs reflowed from the pillars shown in Fig. 1: (a) 450$\mathrm{nm}$ cylindrical, (b) $400-\mathrm{nm}$ square and (c) $600-\mathrm{nm}$ triangular pillars, respectively. The scale bars indicate $1 \mu \mathrm{m}$. After reflow, all nano-SILs exhibit a spherical shape.

Figure 2 shows the 250-nm-height pillars after reflow. The cylinder is transformed into a SIL with diameter $630 \mathrm{~nm}$, the square pillar to a $540-\mathrm{nm}$ SIL, and the triangular pillar to a $630-\mathrm{nm}$ SIL. More detailed quantitative analysis and dimensional characterization is ongoing, and will lead us to the optimal parameters for the hemi-spherical SIL.

\section{OPTICAL CHARACTERIZATION}

In this section, we verify the optical response of the fabricated SILs, which are shown in Fig. 2. The details of the optical characterization are reported elsewhere [6]. We measure the intensity distribution of the focal spot at the bottom of the SIL. For reference, we focus light at the interface between the air and the glass substrate [nonimmersion spot] and compare this with the focal spot on the SIL [immersion spot]. Figure 3 shows the charge-coupled device (CCD) camera images and the intensity distributions of the reference spot and immersion spots through the nanoSILs of Fig. 2. In Fig. 3a, the dark spot in the center indicates the SIL, and the surrounding square is the region where the PMMA has been removed.

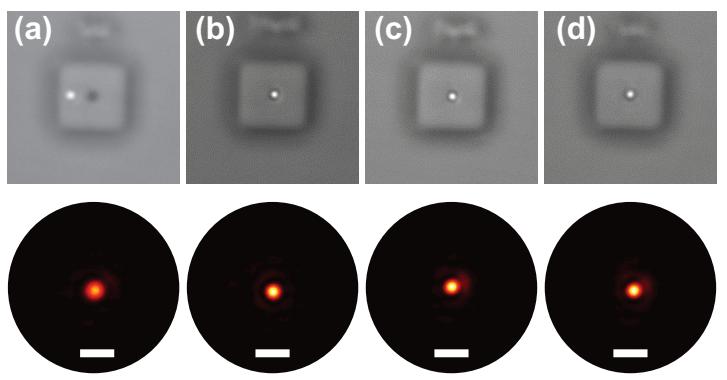

Fig. 3. CCD images and intensity distributions of the focal spots on the bottom plane of the SIL: (a) the reference spot, (b) - (d) the immersion spots through the nano-SILs corresponding to Figs. $2 \mathrm{a}$ $2 \mathrm{c}$, respectively. Upper row: the white spots in each image are the NA0.9 focal spots of the incident light at $642 \mathrm{~nm}$ wavelength. Lower row: the scale bars indicate $1 \mu \mathrm{m}$.
Figures $3 b-3 d$ show smaller focal spots compared to the non-immersion spot of Fig. 3a. Due to their small size, nano-SILs are robust to experimental errors, such as the small variation of dimensions and shape. Therefore, minor variations of the diameter do not strongly affect their performance, and the three nano-SILs shown in Fig. 2 exhibit approximately the same performance in terms of spot-size reduction and enhancement of the peak intensity. The reference spot in Fig. 3a is the NA0.9 focal spot of the illumination at $642-\mathrm{nm}$ wavelength, whose full-width at half maximum (FWHM) is measured to be approximately $400 \mathrm{~nm}$. The FWHM spot size of the immersion spots is measured to be $300 \mathrm{~nm}$ and the peak intensity is $50 \%$ higher. We assume that experimental errors, for example the non-ideal shape of the nano-SILs and the resolution of the measurement system, cause the slight discrepancy from the expected value of the spot size reduction.

\section{CONCLUSIONS}

We demonstrate that reflow and wetting of non-circular shaped nano-pillars can produce spherical structures, which serve as nano-scale SILs. They show the same performance as nano-SILs fabricated from a cylindrical pattern. The fabricated nano-SILs reduce the spot size by a factor of 1.33 and increase by $50 \%$ the measured peak intensity. Investigation of the optimal process parameters, by varying the size of pillars and reflow conditions, is ongoing.

\section{ACKNOWLEDGMENT}

The research leading to these results has received funding from the European Space Agency and the U.S. National Science Foundation.

\section{REFERENCES}

[1] S. M. Mansfield and G. S. Kino, "Solid immersion microscope,” Appl. Phys. Lett. 57, 2615 (1990).

[2] R. Brunner, M. Burkhardt, A. Pesch, O. Sandfuchs, M. Ferstl, S. Hohng, and J. O. White, "Diffraction-based solid immersion lens," J. Opt. Soc. Am. A 21, 1186-1191 (2004).

[3] D. A. Fletcher, K. B. Crozier, C. F. Quate, G. S. Kino, K. E. Goodson, D. Simanovskii, and D. V. Palanker, "Nearfield infrared imaging with a microfabricated solid immersion lens," Appl. Phys. Lett. 77, 2109 (2000).

[4] M. Lang, T. D. Milster, E. Aspnes, T. Minamitani, and G. Borek, "Investigation of micro solid immersion lens mounting systems,” Jpn. J. Appl. Phys. 46, 3737 (2007).

[5] M.-S. Kim, T. Scharf, M. Brun, S. Olivier, S. Nicoletti, and H. P. Herzig, "Advanced optical characterization of micro solid immersion lenses," Proc. of SPIE Vol. 8430, 843012 (2012)

[6] M.-S. Kim, T. Scharf, M. T. Haq, W. Nakagawa, and H. P. Herzig, "Subwavelength-size solid immersion lens," Opt. Lett. 36, 3930-3932 (2011).

[7] A. Schleunitz and H. Schift, "Fabrication of 3D nanoimprint stamps with continuous reliefs using dosemodulated electron beam lithography and thermal reflow," J. Micromech. Microeng. 20, 095002 (2010). 